See discussions, stats, and author profiles for this publication at: https://www.researchgate.net/publication/266618899

\title{
EESOR: Energy Efficient Selective Opportunistic Routing in Wireless Sensor Networks
}

Conference Paper $\cdot$ March 2014

Dol: $10.1007 / 978-3-642-54525-2$

CITATIONS

14

5 authors, including:

Yamuna Devi C R

Dr. Ambedkar Institute of Technology

15 PUBLICATIONS 26 CITATIONS

SEE PROFILE

Venugopal KR

University Visvesvaraya College of Engineering

924 PUBLICATIONS 3,686 CITATIONS

SEE PROFILE
READS

4,043

Discovery of IoT Objects View project

Project Translation Based Face Recognition Using Fusion of LL and SV Coefficients View project

SH Manjula

UVCE, Bangalore University

94 PUBLICATIONS 201 CITATIONS

SEE PROFILE

Lalit M Patnaik

Indian Institute of Science

836 PUBLICATIONS 8,645 CITATIONS

SEE PROFILE 


\title{
EESOR: Energy Efficient Selective Opportunistic Routing in Wireless Sensor Networks
}

\author{
C.R. Yamuna Devi ${ }^{1}$, B. Shivaraj ${ }^{1}$, S.H. Manjula ${ }^{1}$, K.R. Venugopal ${ }^{1}$, and L.M. Patnaik ${ }^{2}$ \\ ${ }^{1}$ Department of Computer Science and Engineering \\ University Visvesvaraya College of Engineering, \\ Bangalore, India \\ ${ }^{2}$ Indian Institute of Science, Bangalore, India \\ yamuna.devicr@gmail.com
}

\begin{abstract}
Opportunistic Routing in wireless sensor networks is a multi-hop routing. In this routing neighbors of a node overhear the transmission and form multiple hops from source to the destination for transfer of information. The set of neighbor nodes participating in the routing are included in the forwarder list in the order of priority. A node with highest priority is allowed to forward the packet it hears. This paper implements Energy Efficient Selective Opportunistic Routing (EESOR), reduces the size of forwarder list by applying a condition that the forwarding node is nearer to the destination. The path followed by acknowledgment packet follows opportunistic routing, assuring reliability of transmission and energy balancing. The simulated results obtained in NS2 simulator show that proposed EESOR protocol performs better than existing Energy Efficient Opportunistic Routing (EEOR) protocol in terms of average End-to-End delay, maximum End-to-End delay and Network Lifetime.
\end{abstract}

Keywords: Delay, Energy, Forwarding, Opportunistic Routing, Wireless Sensor Network.

\section{Introduction}

A wireless sensor network consists of spatially distributed autonomous sensors to cooperatively monitor parameters like temperature, sound, vibration, pressure, motion or pollutants. Sensors are built by recent advances in Micro Electro Mechanical Systems (MEMS) technology. Sensory data comes from multiple sensors in distributed locations in the area where the sensor nodes are deployed. Wireless sensor networks are responsible for sensing and processing the sensed data depending on the requirement of the network. The frequency of sensing by a sensor node may depend on the occurrence of an event or it may be periodical depending on the application for which a node is used.

Industrial application areas where wireless sensor nodes are used include Industrial process monitoring and control machines. Health monitoring, environment and habitat monitoring, health care applications, home automation, and traffic control, are civilian applications of wireless sensor networks. The applications of wireless sensor 
networks demand smaller size for the nodes, and in turn, the components of the nodes. The lifetime of a sensor node depends mainly on the battery contained in the nodes. Because of the wireless nature of nodes, the applications demand long life for sensor nodes. This requires energy of the sensor nodes to be used very efficiently. Applications of wireless sensor networks demand minimum delay in data transmission, good throughput, and longer network lifetime.

Figure 1 shows the block diagram of a wireless sensor node. A typical wireless sensor node has a sensor unit to perform the basic sensing operations, a memory unit to store the sensed data, a battery for power requirements, an embedded processor for data processing and a transceiver for transmitting and receiving the data. The transceiver unit is provided with a limited range antenna. The memory provided in the sensor node is normally a limited storage memory to facilitate small size, as the node is transmitting the data sensed and does not store it. The battery is provided with initial energy and has limited life. With the five necessary units a sensor node can be equipped with optional units such as mobilizer, location finding unit and power generator. Normally, about $50 \%$ of the cost of the sensor node is meant for the cost of the actual sensor unit.

The design of a routing protocol for wireless sensor network is influenced by factors like scalability, fault tolerance, network topology, transmission media, operating environment and power consumption. It is difficult to integrate all these factors into a single network and they are only used as guidelines in the design of a routing protocol. The influencing factors are used for the comparison of different routing schemes for wireless sensor networks. Many applications in wireless sensor networks require information to be transferred between source-destination pairs, that may be one or more hops away.

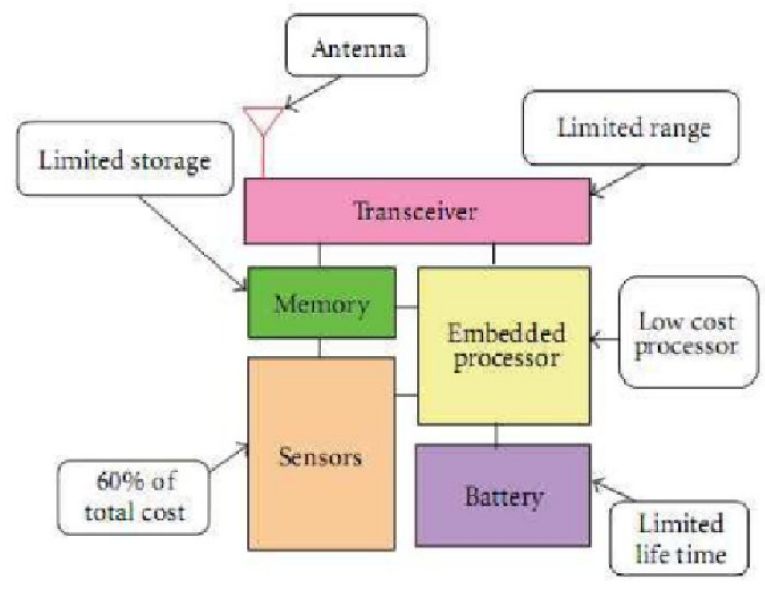

Fig. 1. Wireless Sensor Node Block Diagram

It is an interesting problem to connect the source-destination pairs of a wireless sensor network through the shortest distance, with minimum hops, in a short time with more reliability. When the source and destination are more than one hop away, one of the nodes has to be selected from the set of neighbours of the source to forward 
the packet towards the destination. The nodes in the forwarder list are prioritized based on different metrics like hop count and packet delivery ratio. The choosing of forwarding node continues till the destination node is reached. Different routing protocols are used in disseminating information from source to destination in a wireless sensor network.

Opportunistic routing is one of the flat based reactive routing protocols. Advantages of opportunistic routing protocols are increased reliability and increased transmission range of a node in a wireless sensor network. Network reliability is increased by transmitting a packet through any possible link in the network rather than one specified link. Transmission range is increased by including good quality short-ranged links and poor quality long-ranged links. There are many variants of opportunistic routing, viz, Energy Efficient Opportunistic Routing (EEOR) [1], Exclusive Opportunistic Routing (ExOR) [2], Assistant Opportunistic Routing (AsOR) [3]. Each of the opportunistic routing protocol has its own advantages and disadvantages.

Energy Efficient Opportunistic Routing is a multi-hop routing protocol for wireless sensor networks. It makes use of the forwarders list of the node to choose the forwarding node to transfer the data towards the target. Priorities are assigned for the neighbours of a node to choose the forwarding node. Energy consumption, packet loss ratio, and delivery delay parameters in a wireless sensor network are measured. Efficient protocols are required to reduce delay in transmission and to prolong the network lifetime. EEOR protocol gives better results compared to ExOR protocol in terms of packet loss ratio, average delivery delay and energy consumption. The proposed EESOR protocol achieves better throughput, maximum end-to-end delay and network lifetime, by reducing the size of the forwarder list and opportunistically routing the acknowledgment packet from target to source in the network.

The rest of the paper is organized as follows. In Section 2, we discuss the work related to opportunistic routing. The Background required for the design of a new protocol for wireless sensor network is discussed in Section 3. Section 4, we present the assumptions and implementation details of Energy Efficient Selective Opportunistic Routing. We analyze the newly implemented protocol for different performance parameters in Section 5. Finally, we conclude the paper and discuss future scope in Section 6.

\section{Related Work}

A vast amount of literature is devoted to opportunistic routing in wireless sensor networks. Some of important works are discussed below.

Mao et al., [1] focused on selecting and prioritizing the forwarder list of a node to minimize energy consumption by all the nodes by designing Energy Efficient Opportunistic Routing (EEOR). The network is analyzed for energy consumption, average delivery delay and packet loss ratio. Biswas and Moris, [2] proposed ExOR, to describe an integrated routing and MAC protocol that increases the throughput of multi-hop wireless networks. The protocol chooses each hop destination of a packet's route after the completion of the hop. It gives the choice to decide which of the neighbouring nodes receives the packet. ExOR protocol gives a better throughput 
compared to traditional routing with the same network capacity. Wei et al., [3] discussed Assistant Opportunistic Routing (AsOR) protocol, that is a unicast routing protocol for multi-hop wireless sensor networks. The authors provided a method for the optimal value for the number of nodes in one transmission segment for multi-hop networks.

Bhorkar et al., [4] proposed d-AdaptOR, a distributed, adaptive, and opportunistic routing scheme for multihop wireless ad hoc networks. The advantage of this scheme is optimal performance, with zero knowledge of network topology and channel statistics. The disadvantage of this protocol is ignorance of short-term performance and congestion control in the network. Hsu et al., [5] reviewed the basic concepts of opportunistic routing and describes the components of opportunistic routing. Current trends, issues and challenges in opportunistic routing are explained with examples. The authors discussed the key challenges in opportunistic routing to be addressed by the researchers.

Wang et al., [6] provided a solution for quality transmission, with the combination of link quality variation and broadcasting nature of wireless channels. The solution called CORMAN, gives significant performance improvement over Ad hoc Ondemand Distance Vector routing, with varying mobile settings. Chachulski et al., [7] presented MORE, a MAC-independent opportunistic protocol, that randomly mixes packets before forwarding them. This protocol runs directly on top of 802.11, without the need for any special scheduler. MORE is found to be better than ExOR, with respect to throughput and gain, when there is spatial reuse, both for unicast and multicast. Fang et al., [8] discussed the problems of choosing the opportunistic route to optimize the total utility or profit of multiple simultaneous users in a wireless mesh network. CONSORT, node-CONStrained Opportunistic RouTing, is proposed by combining the primal-dual and subgradient methods. The iterative method reduces the gap between the solutions provided in each iteration and provides the optimal solution, with respect to higher user utilities and profits.

Mazumdar and Sairam, [9] discussed the opportunities and challenges in opportunistic routing, in improving the performance of wireless multi-hop Ad-hoc and wireless sensor networks. Further, the paper outlines several vital design issues that needs to be considered in improving efficiency and deployability of networks. Li et al., [10] proposed the Localized Opportunistic Routing (LOR) protocol that utilizes the distributed minimum transmission selection algorithm to partition the topology into several nested close-node-sets using local information. LOR improves performances over extremely opportunistic routing and MAC-independent opportunistic routing protocol considering the parameters control overhead, end-toend delay and throughput. Rozner et al., [11] developed a model-driven optimization framework to optimize opportunistic routes and rate limits for both unicast and multicast traffic. The authors conducted simulations and test bed experiments to show that the proposed algorithm outperforms shortest path and opportunistic routing protocols. Traffic and topology variations are to be addressed in the future work.

Nasipuri et al., [12] developed algorithms to find the path that consumes minimal energy for node and link-disjoint wireless networks. The performance evaluation shows that the link-disjoint paths consume less energy than node-disjoint paths. The issues related to distributed implementation and optimal centralized algorithms are discussed. Basalamah et al., [13] analyzed opportunistic routing gain under link 
correlation with the loss of data and acknowledgment packets. A new linkcorrelation-aware opportunistic routing scheme is introduced, which exploits the diverse uncorrelated forward links. The simulation work captures the full advantage of opportunistic routing. Levorato et al., [14] have used Multiple-Input MultipleOutput technology, to increase communication parallelism in the network, by multiple concurrent information flows. A cooperative cross-layer scheme is proposed integrating distributed incremental redundancy hybrid automatic retransmission request error control with routing. The scheme to improve the efficiency of transmissions and reduces the interference in the network. Results in the paper show that the network performance is significantly increased.

Zeng et al., [15] gives a comprehensive study on the impacts of multiple rates, interference, and prioritization on the maximum end-to-end throughput and capacity of opportunistic routing. It is shown that opportunistic routing has a higher potential to improve end-to-end throughput. Wang et al., [16] suggested two opportunistic routing algorithms for P2P networks, that exploit the spatial locality, spatial regularity and activity heterogeneity of mobile nodes in a network. Both theoretical analysis and simulation based study reveal that the proposed algorithms outperform the other algorithms in terms of delivery latency and delivery ratio. Shin et al., [17] proposed a parallel opportunistic routing for wireless ad-hoc networks to observe the changes in power, delay and throughput as the number of source-destination pairs increases in the network. A net improvement in overall power-delay trade off is seen as compared to conventional routing since the interference tolerance of receivers is increased in the network.

Passarella et al., [18] investigated the use of a series of opportunistic contacts, in an opportunistic routing environment. The authors implement a scheme for supporting service provisioning in opportunistic networks, and an analytical model for determining the optimal number of parallel executions required to minimize the service time in the network. Myung and Lee [19] proposed a method for avoiding duplicate forwarding of packets in opportunistic routing. The packet includes a small information piggybacked, that reduces the number of repeated packet transmissions, that in turn increases the throughput.

\section{$3 \quad$ Background}

Routing Protocols aim at improving the performance of wireless sensor networks, in terms of the lifetime, the delay and the network throughput. Routing protocols are classified as single hop and multi hop networks, depending on the number of hops to connect the source and target in the network. Based on the network structure routing protocols are classified as flat based, cluster based and location based routing protocols. The establishment of routing path in a wireless sensor network gives reactive and proactive routing protocols. Opportunistic routing is a flat based, reactive, multi-hop routing protocol for wireless sensor networks which applies to both small scale and large scale wireless sensor networks. Opportunistic routing exploits the broadcast nature of wireless sensor networks.

The metrics used for forwarder set selection are hop-count, packet delivery ratio and end-to-end delay. Any one or a combination of these metrics can be used in 
forwarder set selection and for prioritizing the forwarder nodes. Opportunistic routing has potential benefits brought to wireless sensor networks. Challenges faced by opportunistic routing are taken based on network coding coordination, multi-flow rate control, power control with proper bit-rate selection, multi-channel scenario, deployment of nodes and combination of opportunistic routing with selection diversity. Opportunistic routing is analyzed for fixed power model and adjustable power model of wireless sensor network.

ExOR [2] multi-hop routing for wireless sensor networks integrates routing and MAC protocol to increase the throughput of multi-hop wireless sensor networks. ExOR uses long radio links with high loss rates for transfer of data in a network, that usually are avoided in traditional routing. With the same network capacity as traditional routing, ExOR results in high throughput in a multi-hop wireless sensor network. AsOR [3] forwards the data from the source to destination through a sequence of intermediate nodes. The assistant nodes are used to provide protection for unsuccessful opportunistic transmissions. Priority is given to conservation of energy in wireless sensor networks in the implementation of AsOR.

EEOR [1] allows one of the neighbours to participate in the forwarding of the data packets, from source to destination in multi-hop transmissions in a wireless sensor network. The forwarding node is chosen depending on the cost assigned to each of the nodes. To handle the network traffic efficiently, congestion is controlled in the network dynamically adjusting the flow from each source node in the network. Penalty is imposed on the nodes who choose more than one forwarder nodes in multihop transmission. The protocol computes the expected cost for each node and selecting the forwarder list. From the forwarder list the optimal forwarder list is found by opportunistic routing. The proposed EESOR performs better than the existing EEOR in terms of average End-to-End delay, maximum End-to-End delay and network lifetime.

\section{$4 \quad$ Problem Definition}

Wireless sensor network can be single or multi-hop network depending on the transmission range of the sensor nodes. More number of hops increases the delay in transmission and energy consumed by the nodes. The objective of this work is to reduce the energy consumed by the sensor nodes in receiving, transmitting of information and to decrease the delay in transmission of data from source to destination in a wireless sensor network.

The following are the assumptions in the wireless sensor networks considered:

1) The sensor nodes deployed randomly are static.

2) The node density in the network is uniform.

3) All the sensor nodes are deposited with same initial energy.

4) The sensory data generation frequency is uniform.

5) The nodes have same transmission range.

Sending a packet from source to target in a network can be considered to include three parts, viz 1) the source sending the packet to one neighbour node and that node is the target node, 2) if the target is more than one hop away from the source, then there is at least one node in the neighbours list to relay the packet to target, and 
3) agreement on choosing the actual relay node, among the neighbours of the transmitting node. The time and effort incurred achieving the part 1 , is constant. The same for part 2 depends on the distance between the source and the destination. It is very hard to find the cost on coming to an agreement as to choose the relaying node. It is assumed that the overall cost of communication is represented by the distance between the nodes to be communicated in the wireless sensor network. The distance $d$ between two nodes $A(x 1, y 1)$ and $B(x 1, y 2)$ is calculated by the equation,

$$
d=\sqrt{(x 2-x 1)^{2}+(y 2-y 1)^{2}}
$$

Table 1. Network Parameter Notations

\begin{tabular}{|c|l|}
\hline Variable & \\
\hline$N$ & Dumber of the nodes in the network \\
$P$ & number of the packets transmitted between a pair of source and destination \\
$X, Y$ & Maximum value for node's position \\
$T_{r}$ & Transmission Range \\
$n_{x}$ & $x$ co-ordinate of node $n$ \\
$n_{y}$ & $y$ co-ordinate of node $\mathrm{n}$ \\
$d$ & Time taken by a packet in reaching destination from the source node \\
$E_{I}$ & Initial energy of the node \\
$E_{c}$ & Critical energy level of the node \\
$E_{r}$ & Residual energy of the node \\
$T$ & Simulation time \\
\hline
\end{tabular}

Let $s$ represent the source node, $t$ represents the target node and $i_{1}, i_{2}, \ldots, i_{n}$ represent the intermediate nodes in the network. $F(n)$ represents set of forwarder nodes for node $n$. The set of sorted forwarder nodes of $n$ is represented by $F S(n)$. It is obvious that $F S(n)=i_{1}, i_{2}, . ., i_{n}$ probably in a different order. Let $\alpha$ represent the probability that a packet sent by node $s$ is not received by any of the nodes in $F S(n)$. Then $\beta$ represents the probability that a packet sent by node $s$ is received by at least one node in $F S(n)$, which implies the equation

$$
\beta=1-\alpha
$$

The Table 1 shows the list of variables used in the network and their notations. The network scenario can be represented by the mathematical model shown below.

Optimization function is to

$$
\text { Minimize } d
$$

Subject to the following constraints

$$
\begin{gathered}
E_{r}<=E_{I} \text { for all nodes; } \\
E_{r}<=E_{c} ; \text { for all live nodes; } \\
0<=n_{x}<=X ; 0<=n_{y}<=Y ;
\end{gathered}
$$


In the network considered, the source node forms the set of neighbouring nodes to forward the packet, when the destination is more than one hop away from the source. The set of neighbours is sorted according to its distance from the destination, and normally the first of these nodes in the forwarder list relays the packet towards the destination. The procedure continues till the destination node receives the packet. Algorithm Energy Efficient Selective Opportunistic Routing is given in Table 2 This algorithm finds the minimal path between the source and destination pairs specified in the network.

Table 2. Algorithm EESOR: Energy Efficient Selective Opportunistic Routing

Input: Randomly deployed sensor nodes with source and destination pair to be connected.

Output: Path between source-destination pairs with minimal hops.

Step 1: Construct the routing table for all nodes.

Step 2: Form the neighbor list of each source node.

Step 3: Sort it according to ascending order of distance between itself and destination.

Step 4: Relay the data to first node in the sorted list.

Step 5: Update the routing table of the forwarding node.

Step 6: If destination is reached stop else repeat steps 2-4.

Step 7: Transmit acknowledgment towards the source using steps 1-4.

Step 8: Repeat steps 1-5 for all the source nodes in the network.

The fields used in the packet to be communicated are shown below.

1) source

2) packet length

3) packet sequence number

4) $x$ coordinate

5) $y$ coordinate

6) $z$ coordinate

7) distance

8) data

The first field source represents the node that originated the packet. Packet length represents the number of bytes contained in the packet. Packet sequence number is the index of the packet in the overall simulation of the network. $x$ and $y$ coordinates represents the position of the node, and $z$ represents the speed of movement of a mobile node in number of steps per second. As the nodes in our network are static, $z$ coordinate is always 0 . The distance field of the packet represents the geographical distance between the node and the source. The last field is the data to be communicated between the source and the destination nodes. The acknowledgment packet has the same fields, except the data field. 
The routing table of a node consists of the following fields: Destination, Next Hop, Packet Sequence Number and Distance from the node to destination. Each node has a routing table of all its neighbours, consisting of all the required fields. Distance between node and target node is used in updating the routing table entry of the node during multi-hop transmission. Construction of routing table of a node is considered in the following phases. In Phase I, the routing table for all the nodes in the wireless sensor network is constructed. Phase II updates the routing table of the forwarding nodes depending on their distance from the target node in multi hop wireless sensor networks.

\subsection{Phase I - Populating the Routing Table}

Initially, the routing table of every node is constructed based on the neighbouring node information. This phase starts with the construction of a HELLO packet from the node to all its neighbours. Once it is done, a timer is used to broadcast the HELLO packets to all of its neighbours. The HELLO packet is not sent to any particular node. The node that receives packet, checks the packet source field to find out the address of the node that originated the HELLO packet. If the receiver node routing table already has an entry of the source node of the HELLO packet, it drops the packet. Otherwise, it creates a new entry for the node that has sent the HELLO packet with all the necessary fields.

This process of sending HELLO packets and updating the routing table entry, if needed, is continued for all the nodes in the networks have the complete routing table entries. At the end of Phase I, each node is having a routing table for itself, containing all the information of its immediate neighbouring nodes.

\subsection{Phase II - Updating the Routing Table on the Fly}

The routing table constructed in Phase I, is sufficient for communication in a network, if the nodes are one hop distant away only, that is not true always. In Phase II, the entries in the routing table are updated, depending on the position of the source, intermediate nodes and the destination. The distance between the forwarding node and the target node is updated in the routing table entry, so that the next hop node is the one with smallest distance between itself and the target node. According to the concept of Energy-Efficient Selective Opportunistic Routing (EESOR), the next hop to a particular destination is decided on the fly and new protocol implemented is completely opportunistic. This process is repeated till the destination node is reached. At the end of Phase II, we have a shortest path from the source to destination for multi-hop paths in a wireless sensor network.

The Transport Layer Protocol used in this communication is Transmission Control Protocol (TCP). TCP is a reliable protocol, where every packet is guaranteed to be delivered to the destination, by making use of the acknowledgment packet, sent from target to source node. Normally, the acknowledgment packet flows in the reverse path of the data path, using same intermediate nodes. The newly implemented protocol 
Energy Efficient Selective Opportunistic Routing sends the acknowledgment packet opportunistically.

Figure 2 shows an example multi-hop transmission in a wireless sensor network with 10 nodes randomly deployed. Consider the transmission between the sourcedestination node pairs 8 and 3. The existing EEOR protocol takes the path through the intermediate nodes 7,2 and 9, before reaching the destination node. The acknowledgment packet flows in the reverse path. EESOR protocol takes the path through 2 and 9, avoiding node 7, as shown by dark lines and the acknowledgment flows through the nodes 9 and 7, indicated by light lines avoiding the same route as

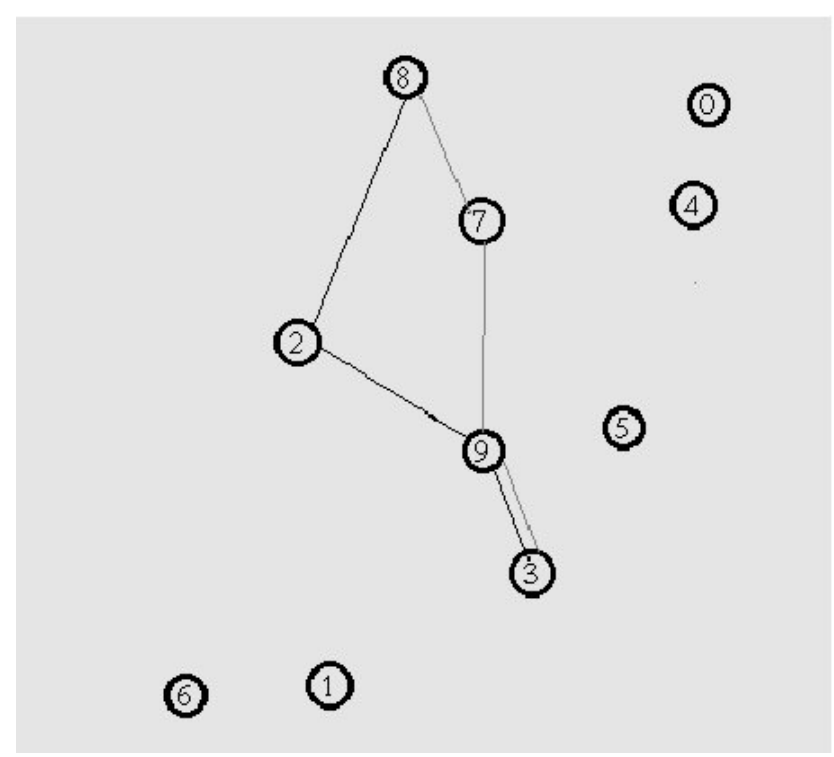

Fig. 2. Energy Efficient Selective Opportunistic Routing for data and acknowledgment packet

the data packet in the Fig. 2. Real time communication involves transmission between different pairs of nodes at different instances of network functioning and the intermediate nodes are chosen according to steps of the algorithm. This real time communication and the use of different paths for the data and acknowledgment packets balances the energy consumed by the nodes in the network and prolongs the network lifetime.

\section{$5 \quad$ Performance Evaluation}

\subsection{Simulation Setup}

The simulator used in the analyzing the wireless sensor network in this paper is NS2. This section provides simulation setup to demonstrate performance of Energy Efficient Selective Opportunistic Routing in the wireless sensor networks. 50 wireless sensor nodes are deployed randomly in a square area of $500 \mathrm{~m}$ by $500 \mathrm{~m}$, with uniform distribution. The packet generation rate is one packet per second. Packets of 1000 
bytes each, are transferred between source and destination pairs for a simulation time of 150 seconds. The acknowledgment packet size is 40 bytes. All the sensor nodes in the network are deposited with an initial energy of 50 Joules. The energy spent by a sensor node in transmission of packet is maximum of 0.38 Joules, in receiving is 0.36 Joules. The node consumes a minimum energy of 0.003 Joules, when it is in idle state.

The behaviour of the network is observed for average End-to-End delay, maximum End-to-End delay and network lifetime. In these 50 nodes, 9 different source- destination pairs are randomly chosen for one-hop, two-hop, and more than two-hop communications. Table 3 shows the details of the nodes connected in the wireless sensor network. The node-pairs 4-41, 43-34, and 39-40 represent one hop communication pairs. The node-pairs 30-32, 10-20, and 1-5 represent two hop communication pairs. The last pairs 0-24, 11-22, and 33-49, represent more than two hops communication source-destination pairs.

Table 3. Details Of Connected Pairs In The Network

\begin{tabular}{|c|c|c|}
\hline Number of Hops & Source Node & Destination Node \\
\hline 1 & 4 & 41 \\
1 & 43 & 34 \\
1 & 39 & 40 \\
\hline 2 & 30 & 32 \\
2 & 10 & 20 \\
2 & 1 & 5 \\
\hline More than 2 & 0 & 24 \\
More than 2 & 11 & 22 \\
More than 2 & 33 & 49 \\
\hline
\end{tabular}

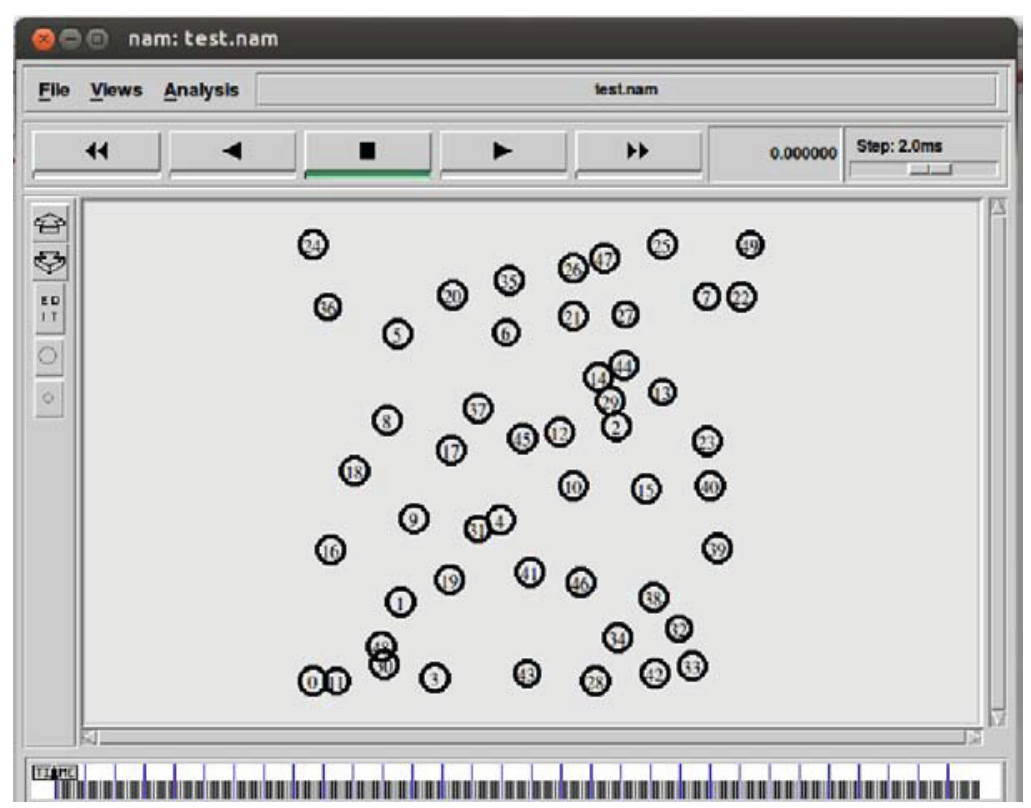

Fig. 3. Network Scenario with 50 Nodes 
For one hop communication, it is not necessary to find the forwarder list and sort it. For two hop communications, the neighbour list of the source has to be formed, prioritized and sorted according to their distance from the target. The node in the forwarder list that is nearer to target is chosen as the forwarding node. For the pairs, $30-32,10-20$ and 1-5, the nodes 28,35 and 8 are used as relaying nodes, respectively. For more than two hop node-pairs, 0-24, the nodes 18 and 36 are used as relaying nodes. For the next pair 11-22, data from 11 goes to node 31, then from node 31 to node 14 , and from 14 it reaches the target 22 . Nodes 40 and 22 are used as relaying nodes in connecting the last pair 33-49.

\subsection{Performance Analysis}

This section analyzes the performance of the wireless sensor network for Energy Efficient Selective Opportunistic Routing for the parameters Maximum End-to-End delay, average End-to-End delay and network lifetime. Fig. 3 shows the network scenario of the wireless sensor network with 50 nodes randomly deployed in the area of $500 \mathrm{~m} \mathrm{X} 500 \mathrm{~m} .250 \mathrm{~m}$ is the transmission range of each of the sensor nodes in the network.

\subsubsection{Average End-to-End Delay}

End-to-End Delay is defined as the time elapsed between the source node sending the packet and the destination node receiving the packet. The average of the End-to-End delay of all the packets transmitted between each of the pairs of source-destinations gives the average End-to-End delay. The average End-to-End delay is plotted against different pairs of source and destinations as shown in Fig. 4.

It is observed that for one hop networks, Energy Efficient Selective Opportunistic Routing does not show any improvement, because the time for choosing the set of forwarder list is not needed. As the number of hops increases, the reduction in delay is more. For two-hop and three-hop communications the delay is reduced up to a maximum of $90 \mathrm{~ms}$ which is the same as $9 \%$ for 10-20 source-destination pair and $295 \mathrm{~ms}$ that is approximately equal to $30 \%$ for $11-22$ source destination pair, respectively.

\subsubsection{Maximum End-to-End Delay}

Figure 5 shows the plot of maximum of End-to-End delay values, for the same 9 pairs of nodes considered for analyzing average End-to-End delay. Once again, single hop communication takes same amount of time in Energy Efficient Selective Opportunistic Routing. Two-hop communication between the nodes 30 and 32 shows the maximum improvement of around $300 \mathrm{~ms}$, or $3 \%$ of total delay for each source destination pair. And more than two-hop communication yields a maximum reduction of delay by approximately $1000 \mathrm{~ms}$, or $50 \%$, for the source destination pair 11-22. The reason for this reduction is decrease in the size of forwarder list in case of Energy Efficient Selective Opportunistic Routing, by considering only the neighbour nodes that are nearer to destination. 


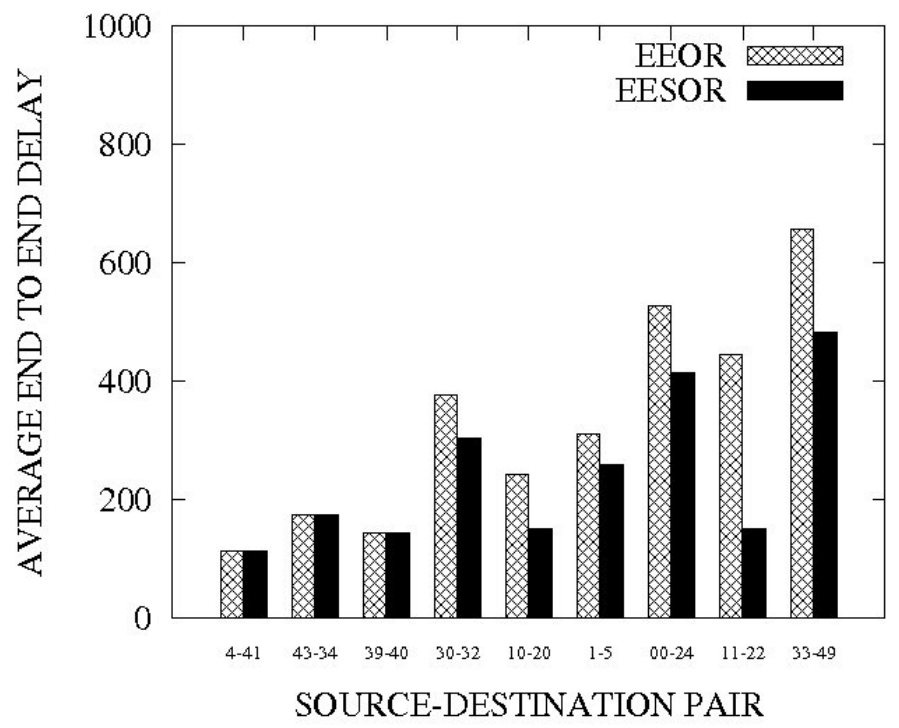

Fig. 4. Average End-to-End Delay

The small size of forwarder list reduces the time taken for prioritizing and sorting the nodes. The time for finding the shortest distance between each node in the forwarder list and the destination is reduced. The analysis of maximum End-to-End delay shows that, as the number of hops increases, the transmission delay increases. The End-to-End delay is lesser in Energy Efficient Selective Opportunistic Routing as compared to Energy Efficient Opportunistic Routing.

\subsubsection{Network Lifetime}

The lifetime of a sensor node is considered as the time from its deployment to the time till which the node is having more than $10 \%$ of its initial energy. The node is said to be alive in this period. Beyond this period the node is said to be dead. Network Lifetime is the time between inception of the network to the time upto which $10 \%$ of the sensor nodes are alive. Fig. 6 shows the network lifetime for both Energy Efficient Opportunistic Routing and Energy Efficient Selective Opportunistic Routing protocols plotted against different network sizes. Network size is considered as 25 nodes, 50 nodes, 75 nodes and 100 nodes for comparing the performance of Energy Efficient Opportunistic Routing and Energy Efficient Selective Opportunistic Routing.

The network performance is analyzed for the network sizes 25, 50,75 and 100 nodes for network lifetime. The graph shows that the network lifetime increases for all the networks considered, irrespective of the network size. The reason is Energy Efficient Selective Opportunistic Routing uses lesser number of hops to reach destination from the source and the acknowledgment packet traverses a path that may not be the same as the data path as compared to Energy Efficient Opportunistic Routing. 


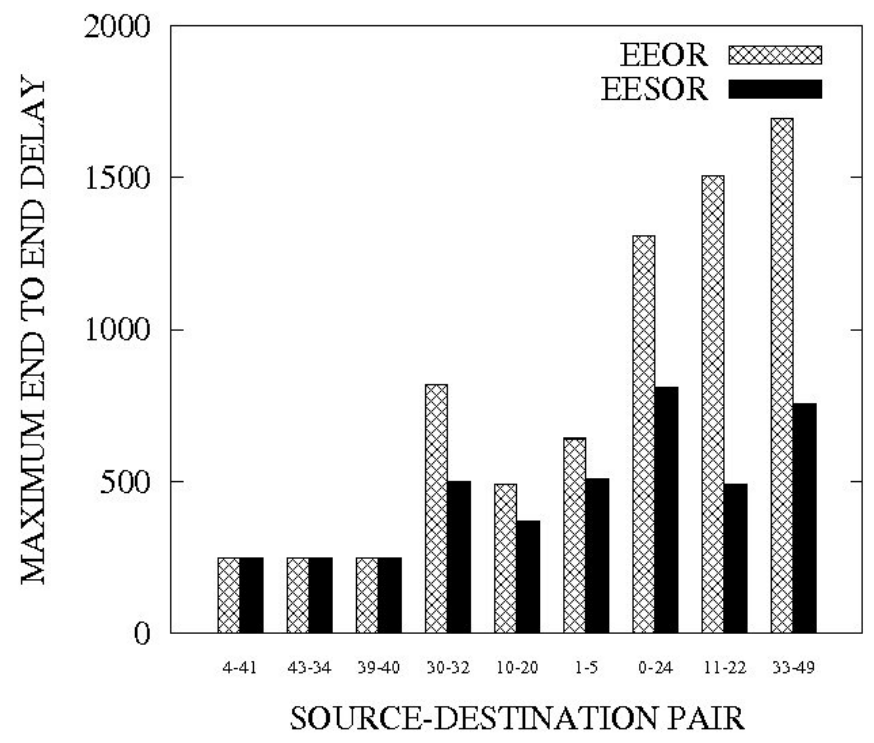

Fig. 5. Maximum End-to-End Delay

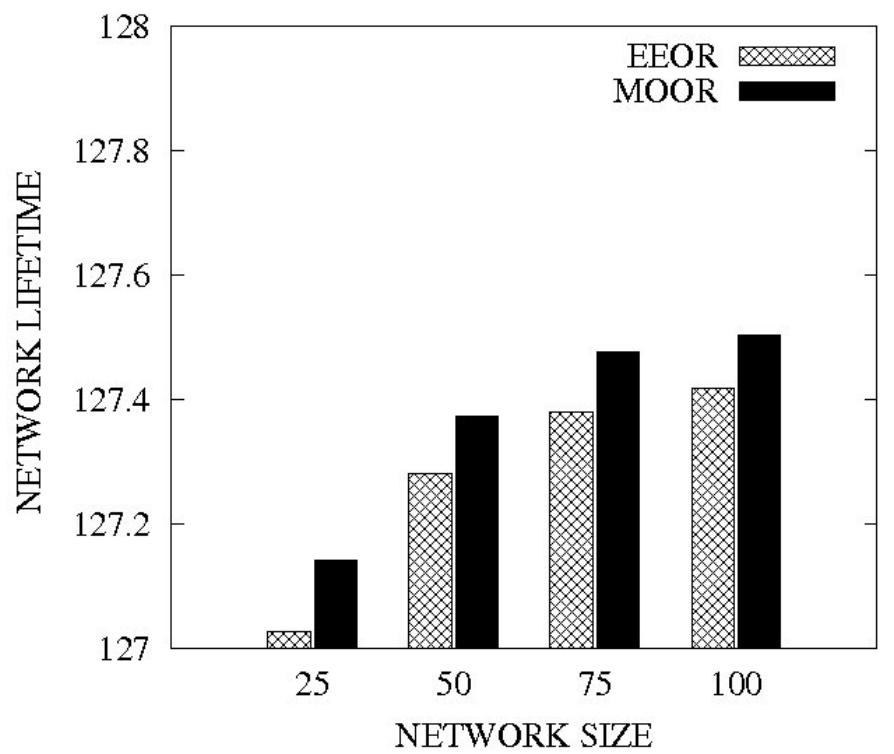

Fig. 6. Network Lifetime 


\section{Conclusions}

In this paper, a novel approach, Energy Efficient Selective Opportunistic Routing is presented, to reduce the average end-to-end delay, maximum end-to-end delay and to increase the lifetime of a multi-hop wireless sensor network. The size of set of forwarder nodes of the source node is reduced by imposing a condition that, the neighbour nodes of the source node nearer to the destination are selected to be included in the set of forwarder nodes. These nodes in the forwarder list are sorted according to the descending order of their distance from the destination node. This technique decreases the average End-to-End delay and maximum End-to-End delay up to maximum of $50 \%$ for some of the source and destination pairs as compared to existing Energy Efficient Opportunistic Routing. Opportunistic routing is applied for the flow of acknowledgment packet from target to source, to balance the energy consumption among the nodes in the network. The lifetime of the network is increased compared to Energy Efficient Opportunistic Routing. The use of Transmission Control Protocol results in the packet delivery ratio to be almost cent percent, as the protocol is reliable. A future enhancement is to analyze Energy Efficient Selective Opportunistic Routing for performance parameters like throughput and turn around time in wireless sensor network.

\section{References}

1. Mao, X.F., Tang, S., Xu, X., Li, X.Y., Ma, H.: Energy Efficient Opportunistic Routing in Wireless Networks. Proceedings of IEEE Transactions on Parallel and Distributed Systems 22(11), 1934-1942 (2011)

2. Biswas, S., Morris, R.: ExOR: Opportunistic Multi-hop Routing for Wireless Networks. In: Proceedings of ACM SICGOMM, pp. 133-144 (2005)

3. Wei, C., Zhi, C., Fan, P., Letaief, K.B.: AsOR: An Energy Efficient Multi-Hop Opportunistic Routing Protocol for Wireless Sensor Networks over Rayleigh Fading Channels. IEEE/ACM Transactions on Wireless Communications 8(5), 2452-2463 (2009)

4. Bhorkar, A.A., Naghshvar, M., Javidi, T., Rao, B.D.: Adaptive Opportunistic Routing for Wireless Ad Hoc Networks. IEEE/ACM Transactions on Networking 20, 243-256 (2012)

5. Hsu, C.-J., Liu, H.-I., Seah, W.K.G.: Opportunistic Routing A Review and the Challenges Ahead. ELSEVIER Journal Computer Networks S5, 3592-3603 (2011)

6. Wang, Z., Chen, Y., Li, C.: CORMAN: A Novel Cooperative Opportunistic Routing Scheme in Mobile Ad Hoc Networks. IEEE Journal on Selected Areas in Communication 30, 289-286 (2012)

7. Chachulski, S., Jennings, M., Katti, S., Katabi, D.: Trading Structure for Randomness in Wireless Opportunistic Routing. In: Proceedings of ACM SIGCOMM (2007)

8. Fang, X., Yang, D., Xue, G.: CONSORT: Node constrained Opportunistic Routing in Wireless Mesh Networks. In: Proceedings of IEEE INFOCOM, pp. 1893-1898 (2011)

9. Mazumdar, A.P., Sairam, A.S.: Opportunistic Routing: Opportunities and Challenges. International Journal Information and Electronics Engineering 2, 247-252 (2012)

10. Li, Y., Mohaisen, A., Zhang, Z.-L.: Trading Optimality for Scalability in Large-Scale Opprotunistic Routing. IEEE Transactions on Vehicular Technology 62(5), 2253-2263 (2013) 
11. Rozner, E., Han, M.K., Qiu, L., Zhang, Y.: Model-Driven Optimization of Opportunistic Routing. IEEE Transactions on Networking 21(2), 594-609 (2013)

12. Nasipuri, A., Castaneda, R., Das, S.R.: Performance of Multipath Routing for On-Demand Protocols in Ad Hoc Networks. ACM/Kluwer Mobile Networks and Applications 6(4), 339-349 (2001)

13. Basalamah, A., Kim, S.M., Guo, S., He, T., Tobe, Y.: Link Correlation Aware Opportunistic Routing. In: Proceedings of 31st Annual International Conference on Computer Communications, pp. 3318-3322 (2012)

14. Levorato, M., Librino, F., Zorzi, M.: Integrated Cooperative Opportunistic Packet Forwarding and Distributed Error Control in MIMO Ad Hoc Networks. IEEE Transactions on Communications 59(8), 2215-2227 (2011)

15. Zeng, K., Lou, W., Zhai, H.: On End-to-End Throughput of Opportunistic Routing in Multirate and Multihop Wireless Sensor Networks. In: Proceedings of INFOCOMM (2008)

16. Wang, S., Cheng, S.: Opportunistic Routing in Intermittently Connected Mobile P2P Networks. IEEE Journal on Selected Areas in Communication 31, 369-379 (2013)

17. Shin, W.-Y., Chung, S.-Y., Lee, Y.H.: Parallel Opportunistic Routing in Wireless Networks. IEEE Transactions on Information Theory 59(10), 6290-6391 (2013)

18. Passarella, A., Kumar, M., Conti, M., Borgia, E.: Minimum-Delay Service Provisioning in Opportunistic Networks. IEEE Transactions on Parallel and Distributed Systems 22(8), 1267-1275 (2011)

19. Myung, J., Lee, W.: Wireless Sensor Networks: A Survey. IEEE Communication Letters 16(4), 510-513 (2012) 